\title{
FLORA ARBUSTIVO-ARBÓREA DE UMA FLORESTA RIPÁRIA NO ALTO RIO GRANDE EM BOM SUCESSO/MG ${ }^{1}$
}

Recebido em 01.09.92. Aceito em 22.08.95.

\author{
Douglas Antônio de Carvalho² \\ Ary Teixeira de Oliveira-Filho ${ }^{3}$ \\ Enivanis de Abreu Vilela ${ }^{2}$ \\ Manuel Losada Gavilanes ${ }^{2}$
}

\begin{abstract}
RESUMO: (Flora arbustivo-arbórea de uma floresta ripária no alto Rio Grande em Bom Sucesso/ MG). Realizou-se o levantamento das espécies com diâmetro ao nível do solo $>=5,0 \mathrm{~cm}$ na mata da Fazenda dos Botelhos, em Bom Sucesso, localizada às margens do Rio Grande, no Sul de Minas Gerais, com o objetivo de reconhecer espécies nativas a serem utilizadas na revegetação das margens de rios e represas da região. Identificaram-se 245 espécies pertencentes à 65 famílias botânicas. São apresentadas comparações florísticas com matas do estado de São Paulo.
\end{abstract}

Palavras-chave: Floresta ripária, Florística

\begin{abstract}
Woody flora of a riparian forest of the ripper Rio Grande, Bom Sucesso, state of Minas Gerais, Brazil). A floristic survey was carried out in a riparian forest of the Rio Grande in Bom Sucesso, Minas Gerais, Brazil, considering the species of trees and shrubs with diameter at the base of the stem $>=5 \mathrm{~cm}$. The purpose was to produce a list of indigenous species for potential use in environmental rehabilitation programs that have bee carried out on the margins of rivers and dams of this region. A list is provided with 245 species of 65 botanic families. Floristic comparisons are made with florests surveyed in the State of São Paulo.
\end{abstract}

Key words: Riparian forest, Floristics

\section{Introdução}

O estudo das matas ciliares no Brasil tem ocupado lugar de destaque nas pesquisas botânicas, tendo em vista a crescente ação devastadora do homem sobre essas que são,

\footnotetext{
' Projeto Integrado Mata Ciliar (Companhia Energética de Minas Gerais: CEMIG) - CONTRATO CEMIG/ESAL/FAEPE.

2 Departamento de Biologia - Universidade Federal de Lavras (UFLA) - Lavras/MG. CEP - 37200-000.

${ }^{3}$ Departamento de Ciências Florestais - Universidade Federal de Lavras - Lavras/MG - CEP 37200-000.
} 
principalmente, as guardiãs dos cursos d'água. A maioria dos estudos existentes foi realizado no estado de São Paulo, notadamente ao longo dos rios Corumbataí (Camargo et al. 1971; Troppmair \& Machado 1974) e Moji-Guaçu (Bertoni \& Martins 1987; Bertoni et al. 1982; Gibbs e Leitão Filho 1978; Gibbset al. 1980; Mantovani et al. 1990).

Em outros estados, como Mato Grosso, Ratter et al. $(1973,1978)$, descreveram as matas de galeria da Serra do Roncador e Oliveira-Filho (1989), Oliveira-Filho \& Martins (1986) e Oliveira-Filho et al., (1990), estudaram a composição florística e a estrutura das matas de galeria da região da Chapada dos Guimarães, associadas a variáveis ambientais. No Distrito Federal, Ratter (1980) estudou matas de galeria da Fazenda Água Limpa. Klein (1980), em Santa Catarina, descreveu a ecologia das matas ciliares do Vale do Itajaí e no Rio Grande do Sul, Bueno et al., (1987), levantaram a flora das margens do Rio Jacuí.

Em Minas Gerais, estudos mais detalhados e específicos sobre comunidades arbóreas de matas ciliares estão ausentes na literatura. Toda a região do Alto Rio Grande, situada no Sul do Estado de Minas Gerais, sofreu ao longo dos anos uma grande devastação de suas matas ciliares. Assim, pela importância sócio-econômica da região e por possuir duas hidrelétricas da Companhia Energética de Minas Gerais (CEMIG), foi estudada neste trecho de rio a composição florística de algumas matas ciliares remanescentes visando a posterior revegetação das margens do rio e das represas com espécies nativas. O presente estudo representa o primeiro de uma série a ser divulgada.

\section{Material e métodos}

\section{Características da área}

O fragmento florestal estudado com área de 84,00 ha, localiza-se na Fazenda Botelho, Distrito de Macaia, Município de Bom Sucesso/MG, próximo do encontro dos Rios das Mortes, Capivari e Grande, sendo este último um dos mais importantes cursos d'agua do sudeste brasileiro (Figura 1). Pode ser caracterizado segundo Veloso et al. (1991) como Floresta Estacional Semidecídua Aluvial (na margem do rio) e Floresta Estacional Montana (no interior da mata). Situa-se numa curva externa do rio em área sujeita a erosão por solapamento, altitude de $825 \mathrm{~m}$, coordenadas $21^{\circ} 9^{\prime} 20^{\prime \prime S}$

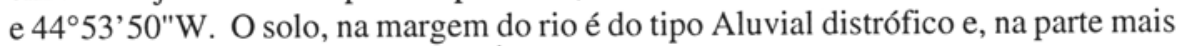
alta da floresta, é um Cambissolo Álico. O clima, de acordo com a classificação de Koeppen, é do tipo Cwb, ou seja, mesotérmico de verões brandos e de invernos secos e amenos. Apresenta temperatura média anual de $19,3^{\circ} \mathrm{C}$, com máximas mensais de $26,9^{\circ} \mathrm{C}$ e mínimas mensais de $14^{\circ} \mathrm{C}$ (Vilela \& Ramalho 1979).

A história do município de Bom Sucesso registra que atividades de garimpo desenvolveram-se entre o final do século XVIII e início do século XIX na área onde existe a mata dos Botelhos. Estes eventos deixaram suas marcas na forma de um labirinto de voçorocas, hoje totalmente recoberto pela floresta. Desta forma, grande parte da mata dos Botelhos resulta de um processo de quase 200 anos de recomposição natural da vegetação. 


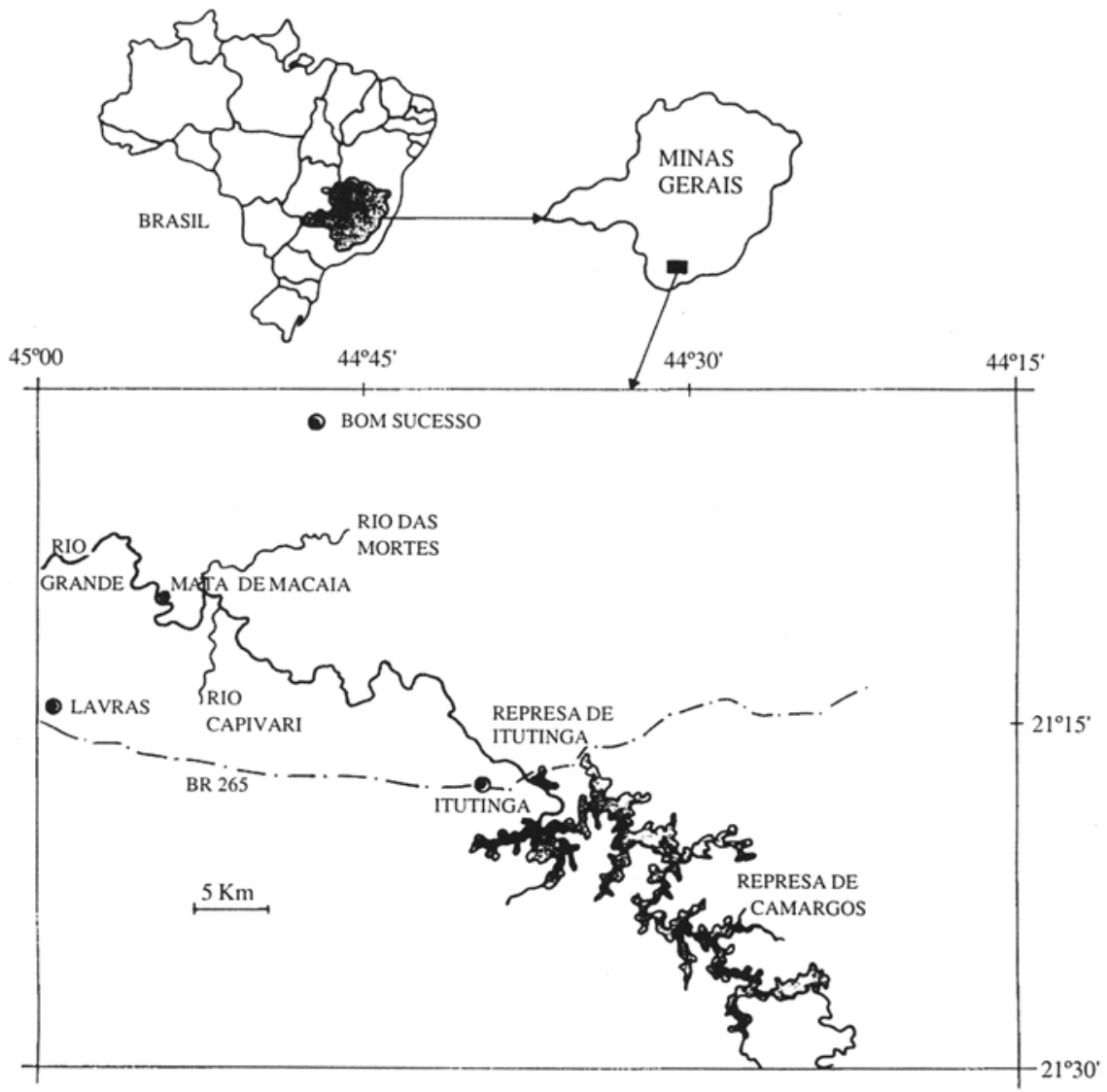

Figura 1. Situação geográfica da Mata de Macaia no estado de Minas Gerais e no Brasil.

\section{Coleta e manuseio do material}

As coletas de material botânico iniciaram-se em março de 1990 e terminaram em março de 1991, através de visitas quinzenais. Os espécimes coletados foram prensados, secos, montados, etiquetados, registrados e incorporados ao Herbário ESAL, do Departamento de Biologia da Universidade Federal de Lavras. Visando a determinação das espécies visitaram-se, ainda, os herbários PAMG(Empresa de Pesquisa Agropecuária de Minas Gerais - EPAMIG - Belo Horizonte/MG), RB (Jardim Botânico do Rio de Janeiro/RJ), SP (Instituto de Botânica de São Paulo, São Paulo/ SP) e UEC (Departamento de Botânica do Instituto de Biologia da Universidade Estadual de Campinas - UNICAMP - Campinas/SP). Quando se fez necessário, as identificações foram feitas ou confirmadas por especialistas. Vários espécimes, em duplicatas, foram doados ao herbário UEC. 


\section{Comparações florísticas}

Foram calculados índices de similaridade de Jaccard (Mueller - Dombois \& Ellenberg 1974) entre o presente levantamento e cinco outros levantamentos florísticos realizados em matas de São Paulo. Estes foram escolhidos em função de sua maior proximidade com a região sul de Minas Gerais e de apresentarem listagens de espécies bastantes expressivas (mais de 130 espécies).

\section{Resultados e discussão}

Foram identificadas 245 espécies, pertencentes a 65 famílias botânicas (Tabela 1). As famílias que apresentaram maiores números de espécies foram: Myrtaceae (30), Lauraceae (18), Fabaceae (17), Euphorbiaceae e Rubiaceae (13), Melastomataceae (9), Annonaceae e Caesalpiniaceae (8), Flacourtiaceae e Meliaceae (7), Mimosaceae (6), Moraceae e Rutaceae (5).

A mata ciliar do presente levantamento apresenta uma similaridade florística, medida pelo coeficiente de Jaccard, de 14 a 24\%, quando comparada com as florestas do Estado de São Paulo, nas regiões mais próximas do sul de Minas Gerais (Tabela 2). Os valores mais baixos foram encontrados nas comparações com as duas florestas semidecíduas montanas que ficam próximas do litoral ou seja, na Serra da Cantareira, em São Paulo (Baitello \& Aguiar 1982) e no Vale do Paraíba, em São José dos Campos (Silva 1989). O valor mais elevado foi encontrado na comparação com a floresta ripária de Moji-Guaçu (Mantovani et al. 1990) e valores intermediários foram obtidos com as duas florestas semidecíduas montanas mais interiores, ou seja, as da Serra do Japi, em Jundiaí (Rodrigues 1986), e da Serra da Mantiqueira, em Atibaia (Meira Neto et al. 1989). Estes resultados indicam que a floresta estudada possui elementos que the conferem características florísticas tanto de mata ciliar como de floresta semidecídua montana. Tais características se associam à sua situação na região serrana sul-mineira e às margens do Rio Grande, sob cuja influência se formou esta floresta.

Tabela 1. Composição florística da mata ripária do Rio Grande, na Fazenda dos Botelho, Distrito de Macaia, Município de Bom Sucesso/MG. As espécies são listadas por famílias e em ordem alfabética e encontram-se acompanhadas de seu nome vernacular e do hábito com que foram encontradas com maior frequência: $\mathrm{A}$ = árvore; $\mathrm{a}=$ arvoreta; $\mathrm{b}=$ arbusto.

ANACARDIACEAE:

Lithraea molleoides(Vell.)Engler Schinus terebinthifolius Raddi

Tapirira guianensis Aublet

Tapirira obtusa (Benth.) Mitchell aroeira-branca, aroeirinha a aroeira-fria, aroeira-vermelha a fruta-de-pombo, pombeiro A pombeiro-branco, pau-pombo A 
Tabela 1. cont.

ANNONACEAE:

Annona cacans Warm.

Duguetia lanceolata A.St.-Hil

Guatteria nigrescens Mart.

Rollinia dolabripetala (Raddi) R.E.Fries

Rollinia laurifolia Schltdl.

Rollinia sericea R.E.Fries

Rollinia sylvatica Mart.

Xylopia brasiliensis Sprengel

$\begin{array}{rc}\text { araticum-cagão } & \mathrm{A} \\ \text { biribá } & \mathrm{a} \\ \text { araticum-seco, pindaíba-preta } & \mathrm{a} \\ \text { araticunzinho } & \mathrm{a} \\ \text { araticum-bravo } & \mathrm{A} \\ \text { araticum-mirim, cortiça } & \mathrm{A} \\ \text { araticum-do-mato, cortiça } & \mathrm{A} \\ \text { pindaíba, pau-de-mastro } & \mathrm{A}\end{array}$

APOCYNACEAE:

Aspidosperma cylindrocarpon Muell. Arg.

Aspidosperma parvifolium A.DC.

Aspidosperma ramiflorum Muell. Arg.

Aspidosperma spruceanum Benth.

Rauvolfia sellowii Muell. Arg.

$\begin{array}{rr}\text { peroba-poca } & \mathrm{A} \\ \text { guatambu, pereiro } & \mathrm{A} \\ \text { guatambu } & \mathrm{A} \\ \text { cabo-de-machado, peroba } & \mathrm{A} \\ \text { casca-d'anta } & \mathrm{a}\end{array}$

ARALIACEAE:

Dendropanax cuneatum (DC.)Decne \& Planchon Schefflera calva (Cham.)D.Frodin

mandioca, maria-mole

\section{ARECACEAE:}

Acrocomia aculeata (Jacquin) Lodd.

Arecastrum romanzoffianum (Cham.) Becc.

Geonoma schottiana Mart.

Syagrus oleracea (Mart.) Becc.

macaúba, bocaiúva

\section{ASTERACEAE:}

Dasyphyllum brasiliense (Spr.) Cabrera

Vernonia discolor Less.

candeia-de-espinho vassourão-preto, cambará

\section{BIGNONIACEAE:}

Cybistax antisyphillitica Mart.

Jacaranda macrantha Cham.

Tabebuia impetiginosa (Mart.) Standl.

Tabebuia serratifolia (Vahl) Nichols

Tabebuia vellosoi Toledo

$\begin{array}{rr}\text { cinco-chagas } & \mathrm{a} \\ \text { caroba-do-mato } & \mathrm{A} \\ \text { ipê-rosa, ipê-roxo } & \mathrm{A} \\ \text { ipê-amarelo } & \mathrm{A} \\ \text { ipê-amarelo } & \mathrm{A}\end{array}$

\section{BLECHNACEAE:}

Blechnum brasiliense Desv. 
Tabela 1. cont.

BURSERACEAE:

Protium almecega Marchand

Protium heptaplyyllum (Aublet( Marchand

Protium widgrenii Engler

\section{CAESALPINIACEAE:}

Bauhinia forficata Link

Bauhinia longifolia (Bongard) Stendel

Cassia ferruginea (Schrad.) Schrad.

Copaifera langsdorffii Desf.

Hymenaea courbaril L.

Peltophorum dubium (Sprengel) Taub.

Sclerolobium rugosum Mart.

Senna multijuga (L.C.Rich.) Irwin \& Barneby

\section{CARICACEAE:}

Jacaratia spinosa (Aublet)A.DC.

\section{CECROPIACEAE:}

Cecropia glazioui Snethl.

Cecropia pachystachya Trécul

\section{CELASTRACEAE:}

Maytenus glazioviana Loesen

\section{CHLORANTHACEAE:}

Hedyosmum brasiliense Mart.

\section{CHRYSOBALANACEAE:}

Hirtella hebeclada Moric.

\section{CLUSIACEAE:}

Calophyllum brasiliense Cambess.

Vismia brasiliensis Choisy

\section{COMBRETACEAE:}

Terminalia glabrescens Mart.

\section{CONNARACEAE:}

Connarus regnellii Schelemberg $\begin{array}{rr}\text { almecega-de-casca-lisa,breu } & \mathrm{A} \\ \text { breu-vermelho } & \mathrm{A} \\ \text { almecega-cascuda } & \mathrm{A}\end{array}$

unha-de-vaca, mororó a

unha-de-vaca, pata-de-boi a canafístula, fedegoso A

copaíba-vermelha, pau-d'óleo A jatobá-d'anta, jataí A angico-cangalha A

angá, cangalheiro, carvoeiro A aleluia, canafístula A

jaracatiá

a

embaúba-vermelha

A

embaúba-cinzenta

A

coração-de-bugre, cafezinho

A

espirradeira-da-mata

b

araçá-da-serra, azeitona

a

guanandi, mangue

A

pau-de-lacre, purga-de-vento

A

carvalho, mirindiba

A

jacaraí

A 
Tabela 1. cont.

CUNONIACEAE:

Lamanonia ternata Vell.

cedrilho

A

CYATHEACEAE:

Cyathea delgadii Sternb.

DICKSONIACEAE:

Dicksonia sellowiana (Presl.) Hook.

xaxim

b

EBENACEAE:

Diospyros inconstans Jacquin

Diospyros hispida A.DC.

fruta-de-jacu

marmelada, olho-de-boi

a

b

ELAEOCARPACEAE:

Sloanea monosperma Vell.

ouriço, castanha-brava

A

ERYTHROXYLACEAE:

Erythroxylum campestre A.St.-Hil.

Erythroxylum cuneifolium (Mart.) Schulz.

Erythroxylum deciduum A.St.-Hil.

fruta-de-tucano, garibaldi fruta-de-pomba

b

fruta-de-pomba

b

b

EUPHORBIACEAE:

Acalypha brasiliensis Muell. Arg. Actinostemon communis (Muell.Arg.) Pax Actinostemon concolor (Sprengel)Muell.Arg. Alchornea glandulosa Poepp. \& Endl. Alchornea triplinervea (Sprengel) Muell.Arg. Croton floribundus Sprengel

Croton urucurana Baillon

Hieronyma ferruginea Tul.

Mabea fistulifera Mart.

Pera obovata Baillon

Sapium longifolium (Muell.Arg.) Huber

Sebastiania brasiliensis Sprengel

Sebastiania schottiana (Muell.Arg.) Muell.Arg.

$\begin{array}{rr}\text { acalifa } & \mathrm{b} \\ \text { laranjeira-brava } & \mathrm{a} \\ \text { pau-rainha, laranjeira-do-mato } & \mathrm{A} \\ \text { tapiá, tanheiro } & \mathrm{A} \\ \text { tanheiro, tapiá-vermelho } & \mathrm{A} \\ \text { tapichingui, capichingui } & \mathrm{A} \\ \text { sangra d'água, sangue-de-drago } & \mathrm{A} \\ \text { sangue-de-boi, quina-do-pará } & \mathrm{a} \\ \text { canudo-de-pito } & \mathrm{a} \\ \text { pau-de sapateiro, cacho-de-arroz } & \mathrm{A} \\ \text { visgueiro, leiteiro } & \mathrm{a} \\ \text { didale, visgueiro } & \mathrm{A} \\ \text { içaranduba, saranduba } & \mathrm{b}\end{array}$

FABACEAE:

Acosmium subelegans (Vogel) Yakovlev

Andira anthelmia (Vell.) Macbr.

Bowdichia virgilioides Kunth

Dalbergia villosa (Benth.) Benth.

Erythrina falcata Benth.

Lonchocarpus guilleminianus (Tul.) Malme

$\begin{array}{rr}\text { sucupira-do-campo } & \mathrm{b} \\ \text { angelim-amargo, pau-se-morcego } & \mathrm{a} \\ \text { sucupira-preta } & \mathrm{A} \\ \text { milho-torrado } & \mathrm{A} \\ \text { mulungu, canivete } & \mathrm{A} \\ \text { embira-de-sapo } & \mathrm{A}\end{array}$


Tabela 1. cont.

Machaerium aculeatum Raddi

Machaerium condensatum Kuhlm. \& Hoehne

Machaerium nictitans Benth.

Machaerium stipitatum (DC.)Vogel

Machaerium villosum Vogel

Myrocarpus frondosus Fr. Allem.

Myroxylon peruiferum L.f.

Ormosia arborea (Vell.) Harms.

Platycyamus regnellii Benth.

Platypodium elegans Vogel

Sesbania sesban (L.) Merr.

\section{FLACOURTIACEAE:}

Casearia arborea (L.Rich.) Urban

Casearia decandra Jacquin

Casearia gossypiosperma Briquet

Casearia lasiophylla Eichler

Casearia sylvestris $\mathrm{Sw}$.

Xylosma ciliatifolium (Clos.) Eichler

Xylosma pseudosalzmanii Sleumer

\section{HIPPOCRATEACEAE:}

Cheiloclinum cognatum (Miers.)A.C. Smith

Salacia elliptica (Mart.) E. Don.

ICACINACEAE:

Citronella paniculata (Mart.) Howard

\section{LACISTEMACEAE:}

Lacistema hasslerianum Chodat

\section{LAURACEAE:}

Aiouea acaradomatifera Kosterm.

Aniba firmula (Nees \& Mart.) Mez

Cinnamomum glaziovii (Mez) Vattino

Cryptocarya aschersoniana $\mathrm{Mez}$

Endlicheria paniculata (Sprengel) Macbr.

Nectandra cissiflora Nees

Nectandra lanceolata Nees

Nectandra megapotamica (Sprengel) Mez

Nectandra nitidula Nees \& Mart.

Nectandra oppositifolia Nees

Nectandra puberula (Schott) Nees

Ocottea catharinensis $\mathrm{Mez}$

Ocotea corymbosa (Meisner) Mez adolfo, jacarandá-de-espinho

jacarandá-braço-de-leão

jacarandá-ferro, bico-de-pato

jacarandá-roxo, sapuva, ximbó jacarandá-mineiro cabreúva, óleo-pardo bálsamo, cabreúva tento, macanaíba pau-pereira, cataguá jacarandá-branco, faveiro capixaba, aleluia

A

A

A

A

A

A

A

A

A

A

b

$\begin{array}{rr}\text { cascaria } & \mathrm{A} \\ \text { canela-espeto, cambroé } & \mathrm{a} \\ \text { japeiro, lingua-de-tiú } & \mathrm{a} \\ \text { espeto-peludo } & \mathrm{a} \\ \text { erva-de-lagarto, guaçatonga } & \mathrm{a} \\ \text { espinho-de-judeu } & \mathrm{a} \\ \text { espinho-de-judeu } & \mathrm{a}\end{array}$

A

a

a

a

a

a

bacupari a

bacupari a

erva-de-anta, assis

A

cafeeiro-do-mato

b

A

canela-sassafrás A

canela-papagaio, garuva A

canela-branca, canela-de-jacu

canela-peluda, canela-do-brejo A

canelão $\quad \mathrm{A}$

canela-cedro A

canela-imbúia A

canela-amarela A

canela-amarela, canela-ferrugem A

louro-amarelo, fruta-de-jacu A

canelinha-preta A

canela-bosta, canela-preta A 
Tabela 1. cont.

Ocotea diospyrifolia (Meisner) $\mathrm{Mez}$

Ocotea laxa (Nees) Mez

Ocotea odorifera (Vell.) Rohwer

Ocotea pulchella (Nees) Mez

Persea pyrifolia Nees \& Mart.

\section{LECYTHIDACEAE:}

Cariniana estrellensis (Raddi) Kuntze

Cariniana legalis (Mart.) Kuntze

Lecythis lanceolata Poiret

\section{LOGANIACEAE:}

Strychnos brasiliensis Mart.

\section{LYTHRACEAE:}

Lafoensia densiflora Pohl

\section{MALPIGHIACEAE:}

Byrsonima laxiflora Griseb.

\section{MELASTOMATACEAE:}

Leandra scabra DC.

Miconia chartacea Triana

Miconia cinnamomifolia (DC.) Naudin

Miconia hispida Cogn.

Miconia ligustroides (DC.) Naudin

Miconia minuflora (Bonpl.) Triana

Miconia pepericarpa DC.

Miconia sellowiana Naudin

Miconia tristis Sprengel

MELIACEAE:

Cabralea canjerana (Vell) Mart.

Cedrela fissilis Vell.

Guarea guidonia (L.) Sleumer

Guarea kunthiana A.Juss.

Trichilia emarginata (Turcz.) C.DC.

Trichilia pallens DC.

Trichilia pallida $\mathrm{Sw}$.

MIMOSACEAE:

Acacia glomerosa Benth.

Albizia polycephala (Benth.) Killip jequitibá-branco, coatinga jequitibá-rosa

sapucaia

A

A

A

solta-martinho

b

dedaleira, mirindiba

a

murici-da-mata

A

pixirica, camará-do-mato b

mexeriquinha a

voadeira, jacatirão A

pixirica a

jacatirão $\quad \mathrm{A}$

pedra-uni, jacatirão b

carvãozinho-vermelho a

jacatirão $\mathrm{A}$

jacatirão A

cangerana, pindaiborana

cedro-rosa, cedro-branco

A

cura-madre, marinheiro guaré, ataúba

catiguá, caá-tigoá

catiguá

catiguá

A

a

A

A

A

A

\section{A} A A 
Tabela 1. cont.

Inga affinis DC.

Inga fagifolia (L.) Willd.

Inga luschnatiana Benth.

Inga striata Benth.

ingá-doce, ingá-miúdo

ingaí a

ingaçu

A

ingá

A

MONIMIACEAE:

Mollinedia widgrenii A.DC.

Siparuna apiosyce (Mart.) DC.

Siparuna guianensis Aublet

pimenteira-brava

A

limoeiro-bravo, folha-santa

negramina, capitiú

a

a

\section{MORACEAE:}

Ficus gomelleira Kunth \& Bouché

Ficus insipida Willd.

Ficus mexiae Standl.

Maclura tinctoria (L.) Don.

Sorocea bonplandii (Baillon) W. Burger

gameleira

figueira

figueira-preta

amoreira, taiúva

canxim, cincho

A

A

A

A

a

MYRSINACEAE:

Myrsine lancifolia Mart.

Myrsine umbellata Mart.

Stylogyne ambigua (Mart.)Mez

pororoquinha-branca

pororoca-branca

pororoquinha-vermelha

A

A

a

\section{MYRTACEAE:}

Blepharocalyx salicifolius (Kunth) Berg

Calycorectes acutatus (Miq.) Toledo

Calyptranthes brasiliensis Sprengel

Calyptranthes chusiaefolia (Miq.) Berg

Calyptranthes lucida Mart.

Campomanesia guazumifolia (Cambess.) Berg

Eugenia aurata Berg

Eugenia blastantha (Berg) Legr.

Eugenia florida DC.

Eugenia involucrata DC.

Eugenia myrtifolia Cambess.

Eugenia pluriflora DC.

Eugenia uniflora L.

Gomidesia affinis (Cambess.) Legr.

Gomidesia eriocalyx (DC.) Legr.

Myrcia laruotteana Cambess.

Myrcia leptoclada DC.

Myrcia multiflora (Lam.) DC.

Myrcia rostrata DC.

Myrcia rufipes DC.

Myrcia tomentosa (Aublet) DC.

Myrcia velutina Berg

Myrcia venulosa DC.

$\begin{array}{rr}\text { guruçuca } & \mathrm{A} \\ \text { amarelinho, grumixama } & \mathrm{A} \\ \text { guamirim } & \mathrm{A} \\ \text { jaborandi } & \mathrm{A} \\ \text { jambo-do-mato, batinga-magra } & \mathrm{A} \\ \text { sete-capotes } & \mathrm{a} \\ \text { murtinha, canzil } & \mathrm{A} \\ \text { casca-de-papel, guamirim } & \mathrm{A} \\ \text { pimenteira } & \mathrm{a} \\ \text { pitanga-preta } & \mathrm{A} \\ \text { pitanguinha-preta } & \mathrm{a} \\ \text { olho-de-boi } & \mathrm{a} \\ \text { pitanga-vermelha } & \mathrm{A} \\ \text { guamirim } & \mathrm{A} \\ \text { guamirim-orelhinha } & \mathrm{a} \\ \text { cocococa } & \mathrm{A} \\ \text { ingabaú } & \mathrm{A} \\ \text { cambui } & \mathrm{A} \\ \text { guamirim-de-folha-miúda } & \mathrm{A} \\ \text { guamirim-lagoa } & \mathrm{a} \\ \text { goiabão, goiabeira-brava } & \mathrm{A} \\ \text { piúna, folha-miúda-cascuda } & \mathrm{A} \\ \text { guamirim-do-campo } & \mathrm{a}\end{array}$

A

A

A

A

A

a

A

A

a

A

a

a

A

A

a

A

A

A

A

a

A

A

a 
Tabela 1. cont.

Myrciaria tenella (DC.) Berg

Psidium cattleianum Sab.

Psidium guajava L.f.

Psidium guineense Sw.

Psidium rufum Mart.

Siphoneugena widgreniana (Berg) Burret

Syzygium jambos (L.) Alston

OCHNACEAE:

Ouratea semiserrata (Mart. \& Nees( Engler

OLACACEAE:

Heisteria silvianii Schwacke

umari

A

OPILIACEAE:

Agonandra engleri Hoehne

cerveja-de-pobre, estoque

a

PIPERACEAE:

Piper aduncum L.

Piper caracolanum S.DC.

Piper dilatatum L.C.Rich

Pothomorphe umbellata (L.) Miq.

POLYGONACEAE:

Coccoloba warmingii Meisner \& Descr.

PROTEACEAE:

Euplassa rufa (Loesen) Sleumer

Roupala brasiliensis Klotzsch

RHAMNACEAE:

Rhamnidium elaeocarpum Reisseck

ROSACEAE:

Prunus brasiliensis (Cham. \& Schltdl.)D. Dietrich

Prunus sellowii Koehne

RUBIACEAE:

Alibertia macrophylla Schum. Amaioua guianensis Aublet

Chomelia sericea Muell.Arg. cabuçu

A

saguaraji-amarelo, tarumaí

a

erva de jaboti, aperta-mão b fruto-de-morcego fruto-de-morcego caapeba-do-norte $\quad b$

catinga-de-barrão carne-de-vaca, catucaém

A

A

pessegueiro-bravo

a

pessegueiro-bravo

marmelada-de-cachorro A marmelada, canela-de-veado veludo-branco

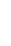

b

A

A

a

A

A

A

a 
Tabela 1. cont.

Coutarea hexandra (Jacquin) Schum.

Faramea cyanea Muell.Arg.

Guettarda uruguensis Cham. \& Schltdl.

Guettarda viburnioides (Cham.) Schltdl.

Ixora warmingii Muell.Arg.

Psychotria barbiflora DC.

Psychotria carthagenensis Jacquin

Psychotria deflexa DC.

Psychotria hastisepala Muell.Arg.

Psychotria sessilis (Vell.) Muell.Arg.

\section{RUTACEAE:}

Esenbeckia febrifuga (A.St.-Hil.) A.Juss.

Galipea multiflora Schult.

Metrodorea stipularis Mart.

Zanthoxylum rhoifolium Lam.

Zanthoxylum tingoassuiba A.St.-Hil.

\section{SAPINDACEAE:}

Allophyllus semidentatus (Miq.) Radlk.

Cupania racemosa (Vell.) Radlk.

Cupania vernalis Cambess.

Matayba jugladifolia (Cambess.) Radlk.

\section{SAPOTACEAE:}

Chrysophyllum gonocarpum (Mart. \& Eichl.)Engler Chrysophyllum marginatum (Hook \& Arn.) Radlk. veludo-preto cafezinho

angélica, veludo-branco jangada, angélica ixora-do-mato erva-de-rato

pau-de-maria, guamirim-preto pau-de-espeto, araçá-bravo pau-se-espeto, araçá-bravo folha-miúda, matadeira

mamoninha, guaxupita guamixinga, grumarim arco-de-pipa, caputuna mamica-de-porca maminha-de-porca

fruta-de-faraó caguatã, camboatã, pavão pau-de-canil, gragoatã caqui-do-mato, cragoatã-branco b

A

a

a

A

b

a

a

b

a

b

a

A

A

A

a

A

A

A

chumbinho, guapeva gumbijava, guatambu-de-leite

A

a

\section{SOLANACEAE:}

Cestrum sendtnerianum Mart.

Sessea regnellii Taub.

STERCULIACEAE:

Guazuma ulmifolia L.

\section{STYRACACEAE:}

Styrax camporum Pohl

Styrax pohli A.DC.

SYMPLOCACEAE:

Symplocos pubescens Klotzsch coeirana

coeirana

b

A

pau-de-motamba, camaçã

A

sabão, benjoim, canelão

A

benjoeiro, salgueiro

A

A 
Tabela 1. cont.

THEACEAE:

Ternstroemia alnifolia Wawra

THYMELAEACEAE:

Daphnopsis brasiliensis Mart. \& Zucc.

Daphnopsis fasciculata (Meisner) Nevl.

\section{TILIACEAE:}

Luehea divaricata Mart. \& Zucc.

Luehea grandiflora Mart. \& Zucc.

Luehea paniculata Mart.

ULMACEAE:

Celtis iguanea (Jacquin) Sargent

Trema micrantha Blume

VERBENACEAE:

Aegiphila lhotzkiana Cam.

Vitex montevidensis Cham

pau-de-papagaio

tarumã

grão-be-galo, joá-miúdo

crindiúva, pau-de-pólvora

b

A

VIOLACEAE:

Hybanthus atropurpureus (A.St.-Hil.)Taub.

ganha-saia

b

VOCHYSIACEAE:

Qualea multiflora Mart.

Vochysia tucanorum Mart.
A

A

A

A

a açoita-cavalo

açoita cavalo 


\section{Referências bibliográficas}

Baittelo, J.B. \& Aguiar, O.T. 1982. Flora arbórea da Serra da Contareira (São Paulo). in: Congresso Nacional Sobre Essencias Nativas, Campos do Jordão, SP, set. 12.18, 1982. Anais... Silvicultura em São Paulo, 16A:582-590 pti (Edição Especial).

Bertoni, J.E.A. \& Martins, F.R. 1987. Composição florística de uma floresta ripária na Reserva Estadual de Porto Ferreira, SP. Acta bot. bras., 1:17-26.

Bertoni, J.E.E.; Stublebine, W.H.; Martins, F.R. \& Leitão Filho, H.F. 1982. Nota prévia: Comparação fitossociológica das principais espécies de florestas de terra firme e da várzea na Reserva Estadual de Porto Ferreira (SP). in: Congresso Nacional Sobre Essencias Nativas, Campos do Jordão, SP, set. 12.18, 1982. Anais... Silvicultura em São Paulo, 16A:563-571 pti (Edição Especial).

Bueno, O.L.; Neves, M.T.M.B.; Oliveira, M.L.A.A.; Ramos, R.L. \& Strehl, T. 1987. Florística em áreas da margem direita do Baixo Jacui, RS, Brasil, Acta bot. bras., 1:101-121.

Camargo, J.C.G.; César, A.L.; Gentil, J.P.; Pinto, S.A.F. \& Troppmair, H. 1971. Estudo fitogeográfico de vegetação ciliar do Rio Corumbatai (SP). Série Biogeográfica n 3. Instituto de Geografia, USP, São Paulo.

Gibbs, P.E. \& Leitão Filho, H.F. 1978. Floristic composition of an area of gallery forest near Mogi-Guaçu, State of São Paulo, S.E. Brazil. Revta. bras. bot., 1(1):151-156.

Gibbs, P.E.; Leitão Filho, H.F. \& Abbott, R.I. 1980. Aplication of the paint-centred quarter method in a floristic survey of the paint-centred quarter method in a floristic survey of an area of gallery forest at Mogi-Guaçu, SP. Brazil. Revta. Bras. bot., 3(1/2):17-22.

Klein, R.M. 1980. Ecologia da flora e vegetação do Vale do Itajaí. Sellowia: v. 32, n. ${ }^{\circ} 32$.

Mantovani, W.; Rossi, L.; Romaniuc Neto, S.; Assad-Ludewigs, I. Y.; Wanderley, M.G.L.; Melo, M.M.R.F. \& Toledo, C.B. 1990. Estudo fitossociológico de áreas de mata ciliar em Mogi-Guaçu, SP,Brazil. in: Simposio sobre mata siliar, Campinas, SP., abr. 11.15, 1989. Anais... p. 235-267 (Fundação Cargill, Campinas).

Meira Neto, J.A.A.; Bermacci, L.C.; Grombone, M.T.; Tamashiro, J.Y. \& Leitão Filho, H.F. 1989. Composição florística da floresta semidecídua de altitude do Parque Municipal da Grota Funda (Atibaia, Estado de São Paulo). Acta bot. bras., 3(2):51-74.

Mueller-Dombois, D. \& Ellemberg, H. 1974. Aims and methods of vegetation ecology: New York, John Wiley \& Sons, 396p.

Oliveira Filho, A.T. 1989. Composição florística e estrutura comunitária de floresta de galeria de córrego da Paciência, Cuiabá, MT. Acta bot. Bras., 3(1):91-112.

Oliveira Filho, A.T. \& Martins, F.R. 1986. Distribuição, caracterização e composição florística das formações vegetais da região da Salgadeira, na chapada dos Guimarães (MT). Revta. bras. bot., 9:207-223.

Oliveira Filho, A.T. Ratter, J.A. \& Shepherd, G.J. 1990. Floristic composition and community structure of a Central Brazilian gallery forest. Flora 184; 103-117.

Ratter, J.A. 1980. Notes on the vegetation of Fazenda Água Limpa (Brasilia, DF, Brazil). Edimburg, Royal Botanic Garden, 169p.

Ratter, J.A.; Richards, P.W. Argent, G. \& Gifford, D.R. 1973. Observations on the vegetation of northeastern Mato Grosso - Expedition area. Philosophical Transactions of the Royal Society of London, B. Biological Sciences, 266:449-492.

Ratter, J.A.; Askew, G.P.; Montegomery, R.F. \& Gifford, D.R. 1978. Observations on forests of some mesophic soils in Central Brazil. Revta. bras. bot., 1:47:58.

Rodrigues, R.R. 1986. Levantamento florístico e fitossociológico das matas da Serra do Japi, Jundiaí, $S P$. Dissertação de mestrado. Campinas, Universidade Estadual de Campinas.

Silva, A.F. 1989. Composição floristica e estrutura fitossociológica do estrato arbóreo da Reserva Florestal Professor Augusto Ruschi, São José dos Campos, SP. Tese de Doutoramento. Campinas, Universidade Estadual de Campinas. 
Troppmair, H. \& Machado, M.L.A. 1974. Variação da estrutura da mata-galeria na bacia do rio Carumbataí (SP), em relação à água no solo, de tipo de margem e do traçado de rio. Série Biogeografia $n^{\circ}$ 8. Instituto de Geografia, USP, São Paulo.

Veloso, H.P.; Rangel Filho, A.L.R.; Lima, J.C.A. 1991. Classificação da vegetação brasileira, adaptada a um sistema universal. IBGE, 123p.

Vilela, E.A. \& Ramalho, M.A.P. 1979. Análise das temperaturas e precipitações pluviométricas de Lavras/MG. Ciência e Prática, 3(1):71-79. 